Revista del Centro de Investigación de la Universidad La Salle

Vol. 14, No. 55, Enero-Junio, 2021: 11-24

DOI: http://doi.org/10.26457/recein.v14i55.2689

\title{
Implicaciones de la obesidad y las enfermedades crónico- degenerativas en las complicaciones por COVID-19: revisión sistemática
}

\section{Implications of obesity and chronic degenerative diseases in complications from COVID-19: a systematic review}

María del Rosario Ayala Moreno Facultad de Ciencias Químicas, Universidad La Salle México (México)

José Carlos Arévalo Sarmiento Facultad de Ciencias Químicas, Universidad La Salle México (México)

Hady Keita

Dirección de Posgrado, Universidad de la Sierra Sur (México)

Dulce María Meneses Ruíz Facultad Mexicana de Medicina, Universidad La Salle México (México)

Tere Hiroko Azures Aguilar Lone Star College North Harris (EEUU)

Carlos Alberto Castañeda López Facultad de Ciencias Químicas, Universidad La Salle México (México) Arely Vergara Castañeda Facultad de Ciencias Químicas, Universidad La Salle México (México)

Recibido: 06 de junio de 2020 Aceptado: 26 de noviembre de 2020

Publicado: 31 de mayo de 2021

\section{Resumen}

El objetivo del presente trabajo fue analizar los reportes que describen la asociación entre algunas comorbilidades como la obesidad y enfermedades crónico-degenerativas (ECD) y las complicaciones por la COVID-19. Se realizó una búsqueda en Pubmed de enero a mayo del 2020 empleando como

Email: rosario.ayala@lasalle.mx

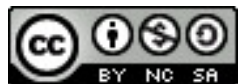

Revista del Centro de Investigación. Universidad La Salle por Dirección de Investigación. Universidad La Salle Ciudad de México se distribuye bajo una Licencia Creative Commons Atribución-NoComercial-CompartirIgual 
Ayala Moreno, M. R.; Arévalo Sarmiento, J. C.; Keita, H.; Meneses Ruíz, D. M.; Azures Aguilar, T. H.; Castañeda López C. A.; Vergara Castañeda, A.

palabras clave obesidad, hipertensión, diabetes, enfermedad crónico-degenerativa, COVID-19, SARSCov2, enzima convertidora de angiotensina y tormenta de citocinas, de la cual se seleccionaron 20 artículos en relación directa con el tema. Se sugiere que aspectos de la fisiopatología de la obesidad y las ECD podrían favorecer las complicaciones del paciente infectado por el virus SARS-COv-2, entre los más descritos hasta el momento, destacan la participación de la enzima convertidora de angiotensina 2 (ECA2) y el desequilibrio inmunológico asociado a la obesidad. En general, el estado proinflamatorio crónico que presenta el paciente con obesidad y el aumento en la expresión de ECA2 en sujetos con hipertensión y diabetes, pueden potenciar el proceso infeccioso incluyendo la incorporación sistémica del virus y favorecer un desequilibrio en la respuesta inmunológica denominado tormenta de citocinas que aumenta el riesgo de mortalidad en el paciente. Si bien el análisis está limitado a escasos reportes, con gran variación en el número de pacientes y cuyos datos descriptivos son sumamente variables, es claro que el analizar la información generada hasta el momento de la actual Pandemia por COVID-19 apoyará en aclarar la participación de la obesidad y la ECD en el proceso fisiopatológico de la enfermedad, sin duda relevante para el manejo terapéutico del paciente especialmente en México, donde las prevalencias de obesidad y ECD son considerablemente elevadas.

Palabras clave: COVID-19, SARS-Cov-2, obesidad, hipertensión, diabetes, enfermedad crónicodegenerativa. 


\section{Abstract}

The aim of this work is to analyze studies related to metabolic comorbidities as obesity and chronic degenerative diseases (CDDs) and COVID-19 complications. A review with Pubmed search until May, 2020 using the keywords obesity, hypertension, diabetes, chronic degenerative diseases, COVID-19, SARS-CoV-2, angiotensin converting enzyme, and cytokine storm, was made. Studies indicate that different aspects related to the pathophysiology of obesity and CDDs promote complication on patient infected with SARS COv-2. In general, the chronic proinflammatory state on obese patient and the increase in the expression of ACE2 in subjects with hypertension and diabetes may enhance the infectious process including the systemic incorporation of the virus and promote higher imbalance in the immune response described as cytokine storm, with higher risk of mortality. Although the analysis is limited to few reports, with higher variation in the number of patients and descriptive data but it is clear that information generated up to the moment on Pandemic by COVID-19 will help to understand relations on obesity and CDDs in relation with complications by COVID-19. These are important for the therapeutic management of the patient, especially in Mexico where the prevalence of obesity and CDDS are considerably higher.

Keywords: COVID-19, SARS-Cov-2, obesity, hypertension, diabetes. 
Ayala Moreno, M. R.; Arévalo Sarmiento, J. C.; Keita, H.; Meneses Ruíz, D. M.; Azures Aguilar, T. H.; Castañeda López C. A.; Vergara Castañeda, A.

\section{Introducción}

En México, de acuerdo a lo reportado por la Encuesta Nacional de Salud y Nutrición (2018) la prevalencia sumada de sobrepeso y obesidad es de $75.2 \%$ en la población (39.1\% sobrepeso y $36.1 \%$ obesidad), siendo las mujeres más afectadas que los hombres (76.8\% vs. $73.0 \%)$, por otra parte, la prevalencia de diabetes diagnosticada es del $10.3 \%$ (11.4\% en mujeres y $9.1 \%$ en hombres), mientras que el $18.4 \%$ de la población tiene hipertensión con cifras ligeramente mayores en mujeres (20.9\%) en comparación con los hombres (15.3\%) (ENSANUT, 2018). Este lamentable panorama proyecta a México como un país con serios problemas de salud pública en lo que respecta a las enfermedades crónico-degenerativas o no transmisibles (UNICEF, 2019; Callahan et al, 2019; ENSANUT 2018; Ayala-Moreno \& Vergara-Castañeda, 2017; Guariguata et al, 2013), lo anterior sugiere que puede presentarse una incidencia mayor de complicaciones en pacientes contagiados por COVID-19, en comparación con otros países del mundo con tasas más bajas de estos padecimientos. La información generada hasta el momento de estudios observacionales es escasa, pero parece sugerir que las comorbilidades metabólicas son un factor de riesgo considerable para las complicaciones por la COVID-19. Todas estas comorbilidades metabólicas tienen en común un estado proinflamatorio y prooxidante, protrombótico y procoagulante que se presenta a lo largo de los años (Acevedo 2006; Ayala-Moreno \& Vergara-Castañeda, 2017), a lo que se suma una interacción con el sistema renina-angiotensina (SRA) que participa de forma importante en el proceso infeccioso por coronavirus y que también se asocia a la fisiopatología de algunas comorbilidades o bien a sus tratamientos (Vaduganathan et. al.,2020; South et. al.,2020; Wan et. al., 2020). Lo anterior sugiere la urgencia de analizar dicha información, que apoye en comprender los mecanismos fisiopatológicos de la enfermedad por COVID-19 y sus complicaciones en el afán de considerar las estrategias que disminuyan el proceso infeccioso y mejoren el manejo terapéutico del paciente con obesidad y ECD.

\section{Métodos}

Se realizó una búsqueda en Pubmed de enero a mayo del 2020 empleando como palabras clave obesidad, hipertensión, diabetes, enfermedad crónico-degenerativa, COVID-19, SARS-Cov2, enzima convertidora de angiotensina y tormenta de citocinas. De dicha revisión se obtuvieron 225 artículos en total, de los cuales de los cuales más el $70 \%$ estaban relacionados con el tema de obesidad y el resto con los enfermedades crónico-degenerativas. De este total fueron seleccionados sólo aquellos que contuvieran el término COVID-19 por asociarse únicamente a la pandemia actual. Dada la poca información que hasta el momento se tenía reportada, se seleccionaron preferentemente artículos de tipo analítico, descriptivo y retrospectivo y solo aquellos que coincidieron en relacionar las complicaciones de la enfermedad COVID-19 con la condición de obesidad y algún enfermedad crónico-degenerativa, teniéndose finalmente un total de 20 artículos en relación estricta con estos temas, a partir de los cuales se deriva la siguiente información. 


\subsection{Coronavirus SARS-Cov-2 y la COVID-19}

El coronavirus SARS-Cov-2 agente causal del síndrome respiratorio agudo severo también denominado COVID-19, posee una estructura constituida de ácido ribonucleico con envoltura monocatenaria. El material genético codifica para cuatro proteínas de membrana, proteínas de espiga (S), de envoltura (E), de membrana $(\mathrm{M})$ y nucleocápside $(\mathrm{N})$. En particular las proteínas "espiga" facilitan su incorporación a la célula huésped (Chan et. al., 2020). La descripción de su genoma recientemente publicada indica que tiene una relación filogenética con los coronavirus del SARS de murciélago y otros SARS humanos como SARS-CoV (Guan et. al., 2020; Chan et. al., 2020); sin embargo, el subdominio externo de la proteína de unión a su receptor esta mutado y presenta solo $40 \%$ de identidad en su secuencia de aminoácidos con otros coronavirus relacionados con el SARS (Chan et. al., 2020).

Derivado de datos observacionales en algunos pacientes, parece ser que los síntomas más comunes de la COVID-19, se presentan con una mediana de 4-5 días (IQR 2-7 días) (Guan et. al., 2020; Wan et. al., 2020) y son fiebre en la gran mayoría de los pacientes (43.8\% al ingreso y $88.7 \%$ durante la hospitalización), tos seca (67,8\%), fatiga (38.1\%), disnea (31.2\%), dolor de cabeza o articular (14.7\%). Otros síntomas pueden ser menos comunes como cambio en la percepción del gusto y olfato, expectoración, dolor de garganta, conjuntivitis, congestión nasal, náusea o vómito, diarrea. La linfocitopenia es un evento que también ha sido reportado en más del $80 \%$ de los pacientes, así como la evidencia radiográfica similar a la neumonía (Guan et. al., 2020; Wan et. al., 2020; De Chang et. al., 2020). La disfunción orgánica, como el shock por dificultad respiratoria aguda, lesión cardíaca aguda y falla renal, han sido relacionados principalmente con el fallecimiento en los casos más severos de la enfermedad (Wan et al, 2020).

Hasta el 7 de mayo según los reportes de la Organización Mundial de la Salud (OMS), se tienen registradas más de 3.5 millones de casos de COVID-19 y 25,000 muertes. Estos reportes estiman 1,626,037 casos con 150,238 muertes en Europa y 1,542,829 casos con 84,804 muertes en América, destacando el mayor número de casos en Estados Unidos (WHO, 2019, datos recuperados semanalmente hasta el 2020). Pese a que la letalidad de COVID-19 es relativamente baja (3.2\%-4.3\%), la alta probabilidad de contagio (Wang et. al., 2020; Guan et. al., 2020) ha favorecido su progresión a pandemia y pone en riesgo la eficiencia de los sistemas de salud, personal y equipo médico en el mundo.

\subsection{Obesidad y enfermedades crónico-degenerativas como factores de riesgo para complicación por COVID-19}

Derivado de estudios observacionales en grupos sobrevivientes y no sobrevivientes infectados con COVID-19, se describieron algunas comorbilidades que parecen ser mucho más prevalentes en pacientes con mayor grado de complicación, su frecuencia varía según el tamaño de la población y los datos clínicos que lograron recopilarse; sin embargo, estos son congruentes en la presencia de hipertensión (15-31.2\%), diabetes (16.2\%-58.3\%) y enfermedades cerebrovasculares (16.7\%-22\%) (Wan et. al., 2020; Guan et. al., 2020; Yang J et. al., 2020; Ynag X et. al., 2020; Fang \& Karakiulakis, 2020). El reporte de Guan et al (2020), parece ser importante por el mayor número de pacientes involucrados $(\mathrm{N}=1099)$, provenientes de 30 provincias de China, cuya característica común fue su diagnóstico con COVID-19 
Ayala Moreno, M. R.; Arévalo Sarmiento, J. C.; Keita, H.; Meneses Ruíz, D. M.; Azures Aguilar, T. H.; Castañeda López C. A.; Vergara Castañeda, A.

bajo condición grave de enfermedad que requiriera su ingreso a una unidad de cuidados intensivos, el uso de ventilación mecánica, o que hubieran fallecido. De este grupo de pacientes, el 23.7\% presentaba hipertensión, $16.2 \%$ diabetes mellitus, $5.8 \%$ enfermedad coronaria y $2.3 \%$ enfermedad cerebrovascular (Guan et. al., 2020). Estos estudios llaman la atención en nuestro país por tener prevalencias alarmantes de estas comorbilidades (obesidad y ECD), que sugieren un posible incremento de los casos de alta complicación en pacientes contagiados con COVID-19. De acuerdo con los resultados preliminares de la última Encuesta de Salud y Nutrición (2018), la prevalencia sumada de sobrepeso y obesidad en población mayor de 20 años en México es de 75.2\% (39.1\% sobrepeso y 36.1\% obesidad), mayor en mujeres que en hombres $(76.8 \%$ vs. $73 \%)$. Por otra parte, otras comorbilidades de mayor importancia asociadas a complicación en pacientes contagiados con COVID-19, son también prevalentes en México, por ejemplo, el porcentaje de la población de 20 años y más con diagnóstico previo de dabetes es del 10.3\% (11.4\% en mujeres y $9.1 \%$ en hombres); mientras tanto, la población con diagnóstico de hipertensión es de $18.4 \%$ (20.9\% en las mujeres y $15.3 \%$ en los hombres) (ENSANUT, 2018).

Existe hasta el momento poca información que pueda apoyar en comprender los mecanismos que establecen la relación entre la obesidad y las ECD con las complicaciones de la COVID-19. Algunos de estos mecanismos están relacionados con el aumento en la expresión de la enzima convertidora de angiotensina 2 (ECA2) y el estado inflamatorio crónico que está presente en pacientes con obesidad y ECD.

En relación al primer mecanismo, los estudios recientes han descrito la participación del sistema renina-angiotensina-aldosterona en la fisiopatología de la COVID-19 (Vaduganathan et al.,2020). Ante esta pandemia, el binomio ECA2 y el sistema renina-angiotensina (SRA) se vuelven un centro de interés para muchos estudios (South et. al., 2020), pues se ha descrito que las proteínas en espiga del virus SARS-Cov-2 poseen un dominio específico de unión a la ECA2 en el huésped, de manera que esta enzima funciona como un receptor viral, y su presencia en diferentes tejidos humanos le confiere al virus la capacidad de infectar a la población (Wan et. al., 2020). La capacidad infecciosa del virus aparentemente puede depender de la densidad de la ECA2 en el tejido y del grado de afinidad entre el dominio de unión de la proteína espiga y secuencias específicas ubicados en la ECA2.

La ECA2 se expresa en diferentes órganos humanos, entre ellos hígado, riñón, intestino, vasos sanguíneos, algunas células inmunes, sistema nervioso central y tejido adiposo, destacando su aumentada expresión en el epitelio alveolar, por tanto, todos estos tipos celulares, pero principalmente el epitelio alveolar (Gheblawi et. al., 2020; South et. al., 2020) que está en contacto más estrecho con el virus en el inicio del proceso infeccioso. De esta manera, todos los órganos que presentan ECA2 son susceptibles de ser infectados por SARS-Cov-2.

La ECA2 es un componente del SRA, el cual está encargado de regular la presión arterial, el volumen sanguíneo y el balance de sodio y potasio. El SRA se activa ante la disminución de la presión arterial o la pérdida de volumen sanguíneo. En dichas situaciones, la renina, una enzima secretada por el aparato yuxtaglomerular en los riñones, es liberada para catalizar la conversión de angiotensinógeno (proteína hepática) en angiotensina I y esta última es convertida a angiotensina II por la ECA. Por otra parte, la ECA2 se encarga de degradar a la angiotensina I y angiotensina II para generar respectivamente angiotensina 1-9 y angiotensina 1-7 (Vaduganathan et. al.,2020; South et. al.,2020). La angiotensina I (precur- 
sor de la angiotensina II) tiene una actividad biológica poco conocida, mientras que la angiotensina II es el vasopresor más potente en nuestro organismo y está presente en distintos tejidos en donde genera diversos efectos como inflamación, fibrosis, estrés oxidativo, vasoconstricción, entre otros (Ponce-Gutiérrez \& Ponce-Gutiérrez, 2012). Por su parte la angiotensina 1-7 tiene funciones antagónicas entre las que destacan vasodilatación y su efecto antiinflamatorio (Figura 1). De este modo, la ECA2 es un regulador fundamental de la actividad de angiotensina II y mantiene un balance en la respuesta inflamatoria y de vasoconstricción (Acevedo, 2006; Ponce-Gutiérrez \& Ponce-Gutiérrez, 2012). Luego entonces, el bloqueo de la ECA2 por presencia de COVID-19 que emplea a esta enzima como receptor, genera una incapacidad de la enzima para catalizar la síntesis de angiotensina 1-7, exacerbando el efecto vasoconstrictor de la angiotensina II por acumulación de esta en el medio (Alifano et. al., 2020; Magrone et. al., 2020).

\section{Figura 1.}

Relación entre el sistema renina-angiotensina-aldosterona y el proceso invasivo del virus SARS-Cov-2 causante de la enfermedad COVID-19

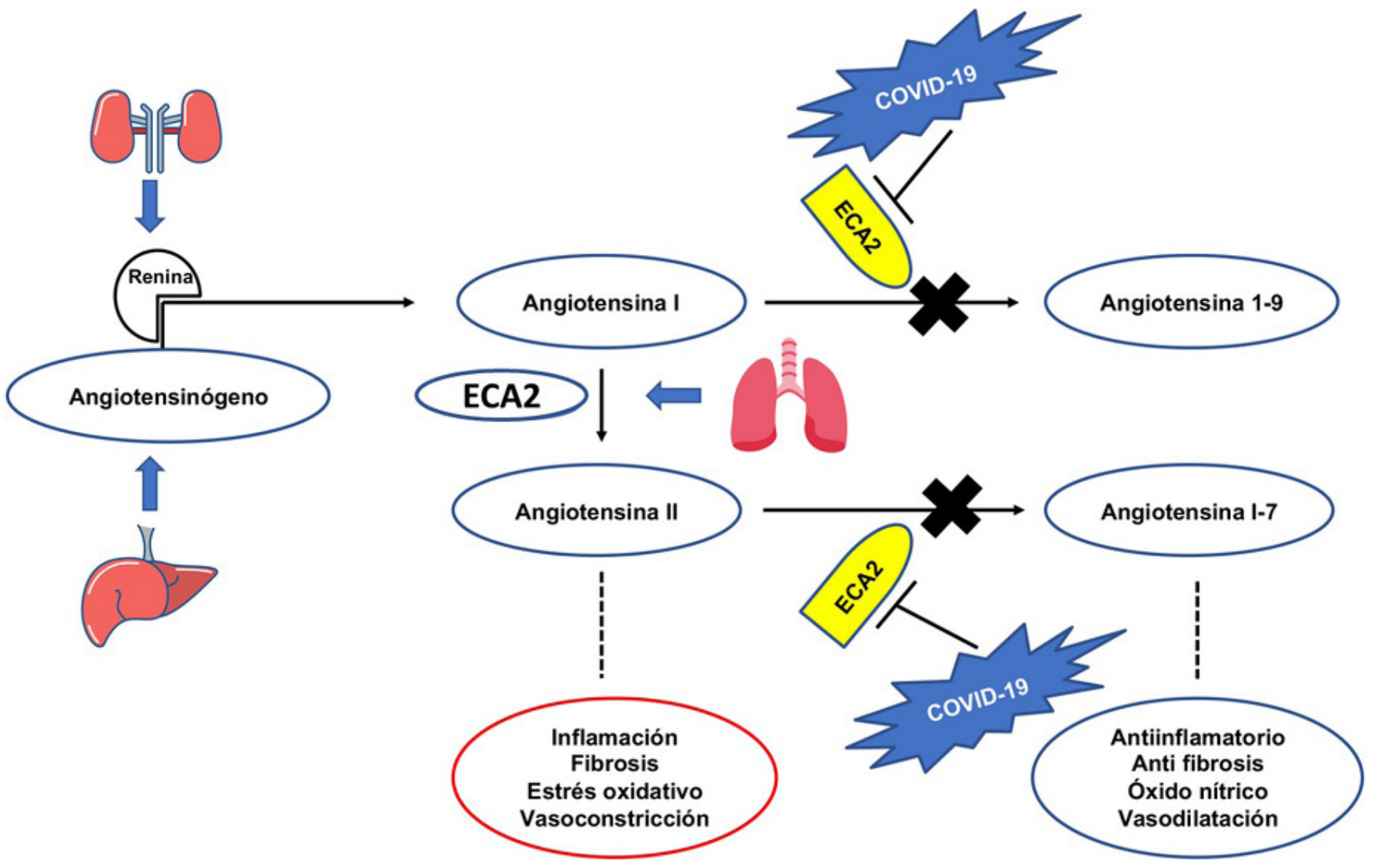

Nota.: ECA: enzima convertidora de la angiotensina; ECA 2: enzima convertidora de la angiotensina 2. El antiotensinógeno de origen hepático es el sustrato de la enzima renina, secretada por las células yuxtaglomerulares del riñón. La renina transforma al Angiotensinógeno en Angiotensina I y éste a su vez se transforma en Angiotensina II por acción de la ECA. La ECA puede ser expresada en el epitelio alveolar. Cuando la ECA esta activa produce Angiotensina II que a su vez se transforma en Angiotesina 1-7 cuyos efectos son antiinflamatorios, antifibrinolíticos, promueve la producción de óxido nítrico y la vasodilatación. Sin embargo, la ECA funciona también como proteína receptora del virus SAR-Cov-2 en cuyo caso pierde su actividad catalítica, 
Ayala Moreno, M. R.; Arévalo Sarmiento, J. C.; Keita, H.; Meneses Ruíz, D. M.; Azures Aguilar, T. H.; Castañeda López C. A.; Vergara Castañeda, A.

promoviendo una acumulación de Antiotensina I, que en consecuencia se transforma en Angiotensina 1-9 con efectos fisiológicos proinflamatorios, relacionados con el mecanismo fisiopatológico de la COVID-19. Fuente: Elaboración propia.

De acuerdo a lo anterior, los factores que favorecen la presencia de ECA2 y angiotensina II podrían aumentar el riesgo de complicaciones en pacientes infectados con COVID-19. Entre estos se han descrito factores ambientales y genéticos. Crackower et. al., (2002) y Solera et. al., (2020), encontraron que los niveles ECA2 están elevados en pacientes con obesidad, hipertensión, dislipidemia, y resistencia a la insulina. Por otra parte, los tratamientos farmacológicos dirigidos a pacientes con diabetes e hipertensión también pueden aumentar la expresión de ECA2 (Alifano et. al., 2020; Fang \& Karakiulakis, 2020). Adicionalmente, algunos polimorfismos de ECA2 se han descrito en sujetos con diabetes e hipertensión en población asiática (Fang \& Karakiulakis, 2020), sugiriendo una interacción de factores ambientales y genéticos que podrían potencializar los riesgos de complicación por COVID-19 en sujetos con obesidad y ECD.

Por otra parte, la obesidad junto con las ECD tiene en común la presencia de un estado de inflamación crónica de bajo grado derivada de un desequilibrio en la producción de citocinas proinflamatorias (Costa de Lucena et. al., 2020). El tejido adiposo está constituido por un conjunto de células entre las que se encuentran infiltradas células entre ellas macrófagos, linfocitos (células asesinas naturales, linfocitos $\mathrm{T}$ cooperadores, linfocitos T reguladores, linfocitos B) y mastocitos, que participan activamente en el desequilibrio de citocinas y quimiocinas característico de la hipertrofia del tejido (Gil et. al., 2011). Desde hace ya algunas décadas se ha descrito que los sujetos con obesidad tienen un deterioro en la capacidad de respuesta de los linfocitos, efectos que son reversibles con la reducción de peso (Tanaka et. al., 1993). Más recientemente algunos estudios han resaltado que la obesidad y las ECD pueden generar alteración de la integridad del tejido linfoide, alteraciones en el desarrollo de leucocitos (fenotipos y actividad), así como en la coordinación de respuestas inmunes innatas y adaptativas. Estos cambios están asociados con un impacto negativo general en la progresión de la enfermedad crónica, la inmunidad contra la infección e incluso sobre la eficacia de la vacunación (Andersen et. al., 2016).

Un evento que se podría relacionar con estas alteraciones inmunológicas de la obesidad y que ha sido parte de las complicaciones con desenlace fatal en los pacientes con COVID-19, es la tormenta de citocinas (cytokine storm), una excesiva respuesta inmunológica al proceso infeccioso de patogénesis compleja, que se ha descrito como una de las mayores causas del síndrome de dificultad respiratoria aguda y falla multiorgánica, que progresan rápidamente generando la muerte del paciente (Ye et. al., 2020)

Lo anterior puede ser fatal para pacientes con obesidad y ECD pues el ambiente proinflamatorio y de estrés oxidativo en concomitancia con el descontrol inmunológico promovido por la infección viral, parecen aumentar de forma importante la tasa de mortalidad (Vaduganathan et. al.,2020; South et. al.,2020; Ponce-Gutiérrez \& Ponce-Gutiérrez, 2012; Costa de Lucena et. al., 2020).

En el caso particular de la Diabetes, se desconoce aún si la enfermedad contribuye de manera independiente al riesgo de desarrollar complicaciones y muerte por COVID-19 considerando que la mayoría de los pacientes diabéticos cursan con una elevada prevalencia de comorbilidades como enfermedad cardiovascular, hipertensión arterial sistémica y obesidad (Yang et. al., 2020). 
El incremento del riesgo de desarrollar complicaciones por COVID-19 en diabéticos está relacionado con diversos mecanismos entre ellos:

1) Incremento en la expresión de ECA2 (Roca-Ho et. al., 2017).

2) Incapacidad de la respuesta inmune para eliminar al virus por la reducción de la actividad quimiotáctica, fagocítica y antimicrobiana de neutrófilos, macrófagos y células asesinas naturales, la deficiente activación de células dendríticas con la consecuente alteración del perfil de citocinas y deficiente activación de linfocitos T (Hodgson et. al., 2015).

3) Mayor susceptibilidad a desarrollar un estado de hiperinflamación y el síndrome de tormenta de citocinas condicionado por el estado de inflamación crónica de bajo grado, secundario al incremento de la formación de productos finales de glicación y a la respuesta tardía de citocinas hiperinflamatorias (Muniyappa \& Gubbi, 2020).

4) Enfermedad cardiovascular preexistente relacionada con el incremento de la expresión de ECA2.

5) Incremento en la expresión de furina, proteasa involucrada en facilitar la infección viral al escindir el dominio S1 y S2 de la proteína espiga en pacientes diabéticos (Figura 2) (Fernández et. al., 2018).

A pesar de que estudios observacionales indican que la edad avanzada y las comorbilidades son factores de riesgo para desarrollar complicaciones por COVID-19, la enfermedad severa también se presenta en pacientes jóvenes aparentemente sanos. Series de casos han documentado el incremento de la relación neutrófilo - linfocito, de niveles séricos de citocinas inflamatorias (IL-6, IL-7, TNF) y quimiocinas (CCL2, CCL3 y CXCL10) y de marcadores inflamatorios como la proteína C reactiva, ferritina y dímeros D, que están relacionados con la gravedad de la enfermedad (Merad \& Martin, 2020).

El perfil de citocinas de pacientes con COVID-19 grave es similar al observado en el síndrome de liberación de citocinas provocado por infecciones severas. El incremento de IL-6 explica en parte la patogénesis de la enfermedad por su efecto pleiotrópico mediante la señalización por el receptor de membrana (mIL-6R), expresado principalmente en hepatocitos, neutrófilos, monocitos-macrófagos y linfocitos B y T activados que contribuye al síndrome de liberación de citocinas. Por otra parte, la señalización de IL-6 mediante su receptor soluble (sIL-6R) en prácticamente todas las células, favorece la activación del endotelio y su participación en la tormenta de citocinas mediante la secreción de proteína quimioatrayente de monocitos-1 (MCP-1), IL-8 e IL-6 adicional. Además, el incremento de secreción del factor de crecimiento endotelial vascular (VEGF), y la reducción de la expresión de E-cadherina en células endoteliales contribuye a la permeabilidad y fuga vascular, implicadas en la fisiopatología de la hipotensión y la disfunción pulmonar por el síndrome de dificultad respiratoria aguda (Ni, et al, 2020) (Figura 2). 
Ayala Moreno, M. R.; Arévalo Sarmiento, J. C.; Keita, H.; Meneses Ruíz, D. M.; Azures Aguilar, T. H.; Castañeda López C. A.; Vergara Castañeda, A.

Figura 2.

Patogénesis de la infección por SARS-CoV-2 y enfermedades crónico-degenerativas.

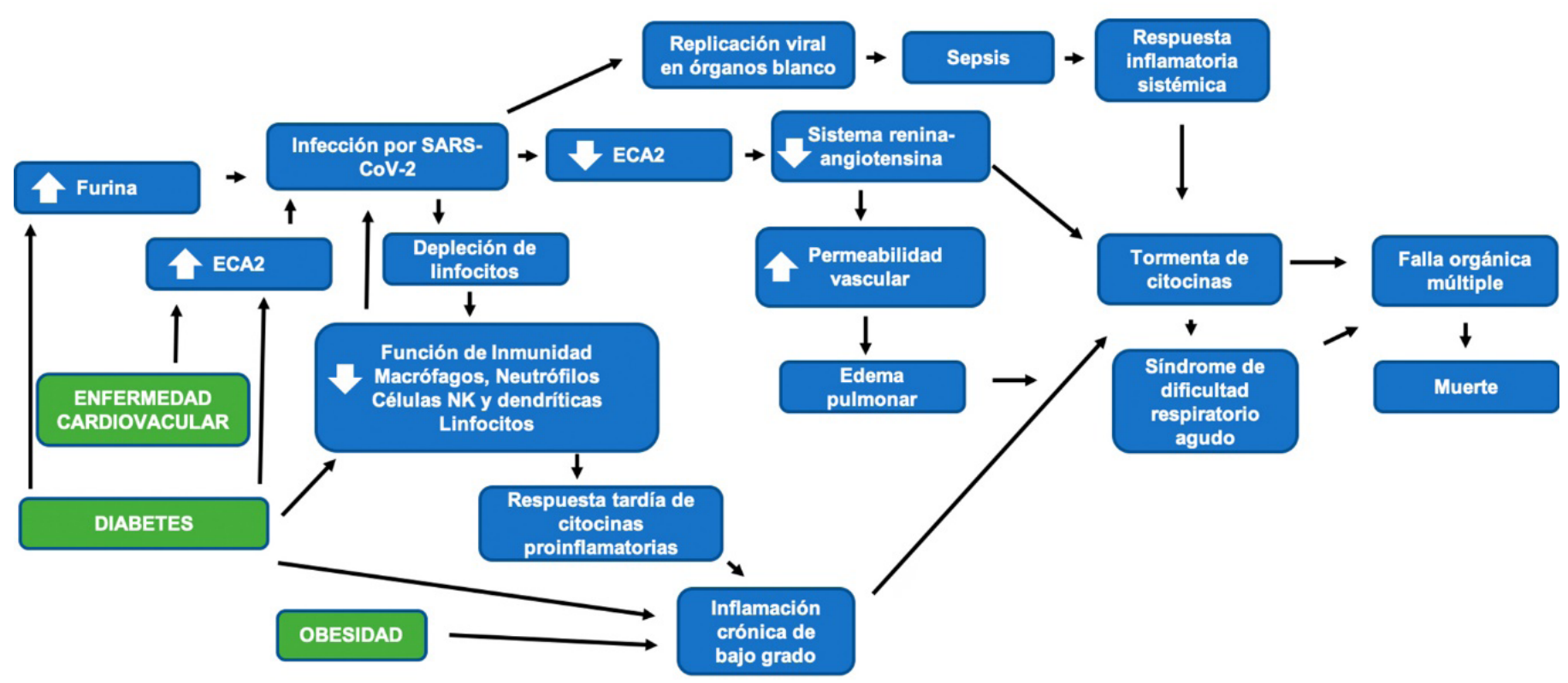

Nota: La infección por SARS-CoV-2 activa la respuesta inmune del huésped que tiene la capacidad de eliminar al virus en la mayoría de los casos. La obesidad, diabetes, hipertensión y enfermedades cardiovasculares predisponen al desarrollo de complicaciones por COVID-19 como el síndrome de dificultad respiratoria aguda, sepsis, falla orgánica múltiple y la muerte. ECA2: enzima convertidora de angiotensina 2. Células NK: células asesinas naturales. Fuente: elaboración propia.

\section{Discusión}

Actualmente, en México estamos sufriendo los estragos de la pandemia y es necesario comprender los mecanismos fisiopatogénicos que están implicados en la enfermedad y sus complicaciones. Hasta el momento, se tienen pocos reportes observacionales que parecen ser constantes en describir una asociación entre la obesidad, diabetes, hipertensión y enfermedad cerebro-vascular y las complicaciones por COVID-19 (Wan et. al., 2020; De Chang et. al., 2020; Fang \& Karakiulakis, 2020; Muniyappa \& Gubbi, 2020; Grasselli et. al., 2020). Sin embargo, hacen falta aún muchos otros estudios que incluyan un mayor número de población y mejor control de las variables que permitan confirmar dichas asociaciones. Pese a ello, el panorama podría ser preocupante en algunos países como el nuestro, en donde las prevalencias de sobrepeso y obesidad (ENSANUT, 2018), así como en ECD son elevadas en comparación con otras regiones del mundo (UNICEF, 2019; Callahan et al, 2019; ENSANUT 2018).

La hipertensión y la diabetes, son actualmente enfermedades que destacan por las complicaciones generadas a largo plazo, causantes de las principales muertes en el país (INEGI, 2019). Estas enfermedades tienen como característica común el estado inflamatorio crónico y estrés oxidativo que de forma 
progresiva deterioran la funcionalidad de diferentes órganos y sistemas (Costa de Lucena et. al., 2020), dando como resultado una vulnerabilidad mayor de estos pacientes a los procesos infecciosos como en el caso de la enfermedad de COVID-19 (Vaduganathan et. al.,2020; South et. al.,2020) Los estudios hasta el momento destacan diferentes aspectos que sugieren algunos mecanismos a través de los cuales la obesidad y las ECD en concomitancia con la COVID-19 pueden ser letales para la población (Wan et. al., 2020; Guan et. al., 2020; Yang J et. al., 2020; Ynag X et. al., 2020; Fang \& Karakiulakis, 2020). Es claro que el estado proinflamatorio, prooxidante y protrombótico característico de la obesidad y algunas ECD, generan un ambiente que favorece la tormenta de citocinas promovida por la COVID-19, acelerando su progresión a síndrome de dificultad respiratoria agua y falla multiorgánica, resultando en la muerte del paciente (Costa de Lucena et. al., 2020). Por otra parte, todos los factores que se asocien con algún mecanismo que favorezca la interacción entre las proteínas S2 y ECA2, pueden no solo aumentar la vulnerabilidad del paciente al proceso infeccioso, si no que determinarían la progresión de la infección a otros órganos que poseen el mismo receptor (ECA2). De modo que tanto los factores ambientales, las comorbilidades como obesidad y ECD, los tratamientos que promueven la expresión del receptor (diabetes e hipertensión) y los factores genéticos (polimorfismos del gen ECA2), deben ponerse en estudio para comprender el riesgo implicado por cada uno de ellos en los pacientes (Alifano et al, 2020; Fang \& Karakiulakis, 2020).

Derivado de este análisis, se lograron identificar un gran número de artículos que asocian las enfermedades crónico-degenerativas y la obesidad con la enfermedad del COVID-19; sin embargo, hasta el momento solamente unos cuantos reportes asocian estrictamente las condiciones de obesidad, diabetes e hipertensión con las complicaciones del COVID-19, lo que limita de cierto modo la presente revisión. Sin embargo, aunque la información es aún escasa, su análisis destaca la importancia de atender el control de la obesidad y las ECD para prevenir o reducir los efectos letales de la COVID-19, pues pese a las cifras oficiales reportadas por la Secretaría de Salud en México, la evidencia científica sugiere que la pandemia en nuestro país puede tener dimensiones catastróficas.

\section{Conclusiones}

Hasta el momento existe unanimidad en la idea de que la obesidad y las enfermedades relacionadas, que componen al síndrome metabólico, constituyen un factor de riesgo importante para las complicaciones derivadas de la infección por coronavirus como el síndrome respiratorio agudo severo SARS.

El estado proinflamatorio y el desequilibrio en la respuesta inmunológica de pacientes con obesidad, así como la descripción de la ACE2 como receptor de SARS-CoV-2, principal responsable del proceso infeccioso en el tejido alveolar y en órganos periféricos, para el desarrollo de la enfermedad por el nuevo coronavirus sugiere un papel importante de la obesidad y las ECD sobre los mecanismos fisiopatológicos en pacientes con mayor complicación por COVID-19. Su estudio y comprensión aún es deficiente y el análisis de la información derivada en futuro será importante para determinar adecuaciones en el tratamiento y manejo clínico de los pacientes, sobre todo en países como México con una prevalencia considerable de estas comorbilidades. 
Ayala Moreno, M. R.; Arévalo Sarmiento, J. C.; Keita, H.; Meneses Ruíz, D. M.; Azures Aguilar, T. H.; Castañeda López C. A.; Vergara Castañeda, A.

\section{Agradecimientos}

Los autores agradecen el apoyo recibido a la Universidad La Salle-Ciudad de México, la Universidad de la Sierra Sur de Oaxaca, México y al Lone Star College, North Harris de Houston Texas, USA.

\section{Fuentes de financiamiento}

El presente trabajo fue apoyado por la Universidad La Salle.

\section{Referencias}

Acevedo, M. (2006). Insulin resistance and hypertension I: mechanisms. Medwave, 6(6), e2305. http:// doi.org/10.5867/medwave.2006.06.2305

Alifano, M., Alifano, P., Forgez, P., Iannelli, A. (2020). Renin-angiotensin system at the heart of COVID-19 pandemic. Biochimie,174, 30-33. http://doi.org/10.1016/j.biochi.2020.04.008

Andersen, C.J., Murphy, K.E., Fernandez, M.L. (2016). Impact of Obesity and Metabolic Syndrome on Immunity. Adv Nutr, 7(1), 66-75. http://doi.org/10.3945/an.115.010207

Ayala-Moreno, M.R., Vergara-Castañeda, A. (2017). Prevalencia del síndrome metabólico en población adulta. En: Panorama global del syndrome metabólico: diagnóstico y epidemiología. Ed. Académica Española (45-77).

Callahan, E.A. (2019). Current Status and Response to the Global Obesity Pandemic: Proceedings of a Workshop. National Academies of Sciences, Engineering, and Medicine; Health and Medicine Division; Food and Nutrition Board; Roundtable on Obesity Solutions. National Academies Press (US) PMID: 31334935 NBK544128. http://doi.org/10.17226/25273

Chan, J.F., Kok, K.H., Zhu, Z., Chu, H., To, K.K., Yuan, S., et. al., (2020). Genomic characterization of the 2019 novel human-pathogenic coronavirus isolated from a patient with atypical pneumonia after visiting Wuhan. Emerg Microbes Infect, 9(1), 221-236. http://doi.org/10.1080/22221751.202 $\underline{0.1719902}$

Costa de Lucena, T.M., Fernandes da Silva, S.A., de Lima, B.R., de Albuquerque, B.M.E., de Azevêdo, S.J. (2020). Mechanism of inflammatory response in associated comorbidities in COVID-19. Diabetes Metab Syndr, 14(4), 597-600. http://doi.org/10.1016/j.dsx.2020.05.025

Crackower MA, Sarao R, Oudit GY, Yagil C, Kozieradzki I, Scanga SE, et al. Angiotensin-converting enzyme 2 is an essential regulator of heart function. Nature, 417(6891):822-828. doi:10.1038/nature00786.

De Chang, Lin M., Wei, L., Xie, L., Zhu, G., De la Cruz, C.S., et. al., (2020). Epidemiologic and Clinical Characteristics of Novel Coronavirus Infections Involving 13 Patients Outside Wuhan, China. JAMA, 323(11), 1092-1093. http://doi.org/10.1001/jama.2020.1623 
Implicaciones de la obesidad y las enfermedades crónico-degenerativas en las complicaciones por COVID-19: revisión sistemática

Encuesta Nacional de Salud y Nutrición (2018). Presentación de resultados. Instituto Nacional de Salud Pública. https://ensanut.insp.mx/encuestas/ensanut2018/doctos/informes/ensanut 2018 presentacion_resultados.pdf

Fang, L. y Karakiulakis, G. (2020). Are patients with hypertension and diabetes mellitus at increased risk for COVID-19 infection? The Lancet, 8, e21. http://doi.org/10.1016/S2213-2600(20)30116-8

Fernandez, C., Rysä, J., Almgren, P., Nilsson, J., Engström, G., Orho-Melander, M., et. al., (2018). Plasma levels of the proprotein convertase furin and incidence of diabetes and mortality. $J$ Intern Med, 284:377-387. http://doi.org/10.1111/joim.12783

Gheblawi, M., Wang, K., Viveiros, A., Nguyen, Q., Zhong, J.C., Turner, A.J., et. al., (2020) Angiotensin-Converting Enzyme 2: SARS-CoV-2 Receptor and Regulator of the Renin-Angiotensin System: Celebrating the 20th Anniversary of the Discovery of ACE2. Circ Res, 126(10), 1456-1474. http://doi.org/10.1161/CIRCRESAHA.120.317015

Gil, A,, Olza, J., Gil-Campos, M., Gómez-Llorente, C., Aguilera, C.M. (2011). Is adipose tissue metabolically different at different sites? Int J Pediatr Obes, 6, 13-20.

Grasselli, G., Zangrillo, A., Zanella, A., Antonelli, M., Cabrini, L., Castelli, A., Cereda, D., Coluccello, A., Foti, G., Fumagalli, R., Iotti, G., Latronico, N., Lorini, N., Merler, S., Natalini, G., Piatti, A., Ranieri, M.V., Scandroglio, A.M., Storti, E., Cecconi, M., Pesenti, A. (2020). Baseline Characteristics and Outcomes of 1591 Patients Infected With SARS-CoV-2 Admitted to ICUs of the Lombardy Region, Italy. JAMA, 323(16), 1574-1581. http://doi.org/10.1001/jama.2020.5394

Guan, W., Ni, Z., Hu, Y., Liang, W., Ou, Ch., He, J., et. al., (2020) Clinical characteristics of coronavirus disease 2019 in China. N Engl J Med, 382, 1708-1720. http://doi.org/10.1056/NEJMoa2002032

Guariguata, L., Whiting, D.R., Hambleton, I., Beagley, J., Linnenkamp, U., Shaw, J.E. (2013) Global estimates of diabetes prevalence for 2013 and projections for 2035. IDF Diabetes Atlas. Diabetes Res Clin Pract, 103(2), 137-49. http://doi.org/10.1016/j.diabres.2013.11.002

Hodgson, K., Morris, J., Bridson, T., Govan, B., Rush, C., Ketheesan, N. (2015). Immunological mechanisms contributing to the double burden of diabetes and intracellular bacterial infections. Immunology, 144, 171-185. http://doi.org/10.1111/imm.12394

Instituto Nacional de Estadística y Geografía (INEGI). Reporte de casuas de mortalidad 2019. https:// www.inegi.org.mx/contenidos/saladeprensa/boletines/2019/EstSociodemo/

Magrone, T., Magrone, M. y Jirillo, E. (2020). Focus on Receptors for Coronaviruses with Special Reference to Angiotensin-converting Enzyme 2 as a Potential Drug Target - A Perspective [Epub ahead of print]. Endocr Metab Immune Disord Drug Target,. http://doi.org/10.2174/1871530320666200 $\underline{427112902}$

Merad, M. y Martin, J.C. (2020). Pathological inflammation in patients with COVID-19: a key role for monocytes and macrophages. Nat Rev Immunol, 20, 355-362. http://doi.org/10.1038/s41577-020$\underline{0331-4}$

Muniyappa, R. y Gubbi, S. (2020). COVID-19 Pandemic, Coronaviruses, and Diabetes Mellitus. Am J Physiol Endocrinol Meta, 318(5), E736-E741. http://doi.org/10.1152/ajpendo.00124.2020 
Ayala Moreno, M. R.; Arévalo Sarmiento, J. C.; Keita, H.; Meneses Ruíz, D. M.; Azures Aguilar, T. H.; Castañeda López C. A.; Vergara Castañeda, A.

Ni, L,. Ye, F., Cheng, M.L., Feng, Y., Deng, Y.O., Zhao, H., et. al., (2020). Detection of SARS-CoV-2Specific Humoral and Cellular Immunity in COVID-19 Convalescent Individuals. Immunity, S2,17. http://doi.org/10.1016/j.immuni.2020.04.023

Organización Mundial de la Salud (2020). Coronavirus disease (COVID-19) Pandemic. Weekly Epidemiological and Operational updates September 2020. https://www.who.int/emergencies/diseases/ novel-coronavirus-2019

Ponce-Gutiérrez, Y., Ponce-Gutiérrez, A. (2012). El sistema renina-angiotensina desde la circulación hasta la célula: implicaciones más allá de la hipertensión. CorSalud (Revista de Enfermedades Cardiocasculares) 4(4), 287-293.

Roca-Ho, H., Riera, M., Palau, V., Pascual, J. y Soler, M.J. (2017). Characterization of ACE and ACE2 Expression within Different Organs of the NOD Mouse. Int J Mol Sci, 18(3), 563. http://doi. org/10.3390/ijms18030563

South, A.M., Diz, D.I. y Chappell, M.C. (2020). Perspectives integrative cardiovascular physiology and pathophysiology covid-19, ACE2, and the cardiovascular consequences. Am J Physiol Heart Circ Physio, 318, H1084-H1090. http://doi.org/10.1152/ajpheart.00217.2020

Tanaka, S., Inoue, S., Isoda, F., Waseda, M., Ishihara, M., Yamakawa, T., et. al., (1993). Impaired immunity in obesity: suppressed but reversible lymphocyte responsiveness. Int J Obes Relat Metab Disord, 17(11), 631-636.

UNICEF, México. Informe: Sobrepeso y obesidad en niños y niñas y doalescentes. https://www.unicef. org/mexico/sobrepeso-y-obesidad-en-ni $\% \mathrm{C} 3 \% \mathrm{~B} 1$ os-ni $\% \mathrm{C} 3 \% \mathrm{~B} 1 \mathrm{as}-\mathrm{y}$-adolescentes

Vaduganathan, M., Vardeny, O., Michel, T., McMurray, J.V.J., Pfeffer, M.A., et. al., (2020). Renin-Angiotensin-Aldosterone System Inhibitors in Patients with Covid-19. N Engl J Med, 382(17), 1653 1659. http://doi.org/10.1056/NEJMsr2005760

Wan, Y., Shang, J., Graham, R., Baric, R.S. y Lia, F. (2020). Receptor Recognition by the Novel Coronavirus from Wuhan: an Analysis Based on Decade-Long Structural Studies of SARS Coronavirus. $J$ Virol, 94(7), e00127-20. http://doi.org/10.1128/JVI.00127-20

Wang, D., Bo, H., Hu, Ch., Zhu, F., Liu, X., Zhang, J., et. al., (2020). Clinical Characteristics of 138 Hospitalized Patients With 2019 Novel Coronavirus-Infected Pneumonia in Wuhan, China. JAMA, 323(11), 1061-1069. http://doi.org/10.1001/jama.2020.1585

Yang, J., Zheng, Y., Gou, X., Pu, K., Chen, Z., Guo, Q., et. al., (2020). Prevalence of comorbidities in the novel Wuhan coronavirus (COVID-19) infection: a systematic review and meta-analysis. Int $\mathbf{J}$ Infect Dis, 94, 91-95. http://doi.org/10.1016/j.ijid.2020.03.017

Yang, X., Yu, Y., Xu, J., Pu, K., Chen, Z., Guo, Qinghong., et. al., (2020). Clinical course and outcomes of critically ill patients with SARS-CoV-2 pneumonia in Wuhan, China: a single-centered, retrospective, observational study. Lancet Respir Med 2020, Feb 24. http://doi.org/10.1016/S2213$\underline{2600(20) 30079-5}$

Ye, Q., Wang, B., Mao, J. (2020). The pathogenesis and treatment of the 'Cytokine Storm' in COVID-19. J Infect, 80(6), 607-613. http://doi.org/10.1016/j.jinf.2020.03.037 